\title{
Central Corneal Thickness - How Should We Interpret the Findings in Children?
}

\author{
John L Brookes \\ Glaucoma Service, Moorfields Eye Hospital, London, UK
}

The measurement of central corneal thickness (CCT) in the management of adults with suspect glaucoma, or established glaucoma has become routine practice. A thick CCT is thought to result in a falsely elevated intraocular pressure (IOP). Conversely, a thinner CCT is thought to result in an under-reading of the IOP. Put simply, a thinner CCT requires less force to applanate it, causing an underestimate of the 'true' IOP. Nomograms and CCT calculators have been devised to simply adjust for the CCT and give a 'true' IOP reading, and to determine the risk of developing glaucoma. Is this too simplistic in adult patients and how does this relate to paediatric corneas and how we take account of CCT in the management of paediatric glaucoma?

In 2002, the Ocular Hypertension Treatment Study (OHTS) showed that CCT was an important predictive factor for the development of glaucoma in adults. ${ }^{1}$ In a multivariate model, CCT was the most powerful component of the predictive model. Furthermore, in a merged European Glaucoma Prevention Study/ OHTS analysis, a reduced CCT measurement was associated with an increased risk of developing glaucoma and a thin CCT was a significant risk factor for progression of glaucoma in patients with a higher baseline IOP. ${ }^{2}$ In this study, each $40 \mu \mathrm{m}$ of CCT reduction conferred a 2 -fold increased risk of developing glaucoma over 5 years (hazard risk, 2.04; 95\% confidence interval, 1.70-2.45).

The question remains how the CCT influences the risk of glaucoma; is it simply related to the accuracy of tonometry, where Goldmann applanation tonometry is designed to give accurate readings of IOP with a CCT of 500 to $520 \mu \mathrm{m}$ ? Alternatively, is CCT linked to the underlying pathophysiology of glaucoma and could it be a marker for the structural integrity of the lamina cribrosa, for example? The Early Manifest Glaucoma Trial results provide some evidence from a clinical trial that the role of CCT in determining glaucoma risk may derive from more than just its influence as an artefact of Goldmann tonometry. ${ }^{3}$

Correspondence: Dr John L Brookes, Glaucoma Service, Moorfields Eye Hospital, 162 City Road, London EC1V 2PD, UK.
Although the role of CCT in adults has been studied for many years, we are just beginning to examine the role of CCT in children. The average CCT in children reaches adult levels by the age of 3 years, and there is little reported effect of age on CCT. Race appears to affect the CCT, and Muir et al ${ }^{4}$ and Dai and Gunderson ${ }^{5}$ have shown that Afro-Caribbean children have a thinner CCT than white children.

In primary congenital glaucoma, CCT is lower than average, which may reflect the larger and stretched corneas in many of these children without corneal oedema. Other subgroups of children with glaucoma such as those with Down syndrome, ${ }^{6}$ Marfan syndrome, ${ }^{7}$ and osteogenesis imperfecta ${ }^{8}$ have also been reported to have a thinner than average cornea. In addition, children who are aphakic or pseudophakic have been consistently shown to have a thicker cornea than average, ${ }^{9}$ and this appears to occur after surgery and also increases over time. CCT before congenital cataract surgery tends to be normal when compared with controls and also compared with fellow non-cataractous eyes. The aetiology of this increased CCT remains unclear, as does how we should use this information in our management of the condition.

Brandt first reported an increased corneal thickness in congenital aniridia, ${ }^{10}$ and a report in this issue by Agarwal et al from the Aravind Eye Hospital in India also shows an increased CCT in children with aniridia in an Indian population. ${ }^{11}$ The study aimed to determine the range and distribution of CCT in aniridia, compared with an age- and sex-matched control group. The average CCT in the aniridia group was $0.603 \mathrm{~mm}$ and in the control group was $0.566 \mathrm{~mm}(p=0.0083)$. Based on the assumption that approximately $50 \%$ of children with aniridia will develop glaucoma and most of these children will require surgery, the authors recommend that CCT should be considered when making decisions on the accuracy of IOP measurements and, hence, decisions on the treatment required. However, we need to be very careful about making management plans and placing too much emphasis on CCT measurements in children. Much work needs to be done on corneal biomechanics, especially as certain groups of children with glaucoma with an increased CCT, such as those with aphakia and aniridia, are those who are at increased risk for glaucoma anyway. 
An abnormal composition of the cornea or the effects of corneal hysteresis may alter our perceived notions of how CCT affects IOP measurements in children. 'Adjusting' for CCT in children needs to be avoided, and we must not overestimate the value of the CCT measurements until further work on corneal characteristics in children is carried out.

In adults, there is increasing evidence that CCT may be linked to glaucoma risk, but there is still much work to be done. Measuring CCT in children gives us some interesting information and we should continue to collect this data, but its value at the present time must not be overestimated. Management decisions should still be based on signs of glaucoma progression. Further studies on corneal hysteresis and other corneal characteristics, rather than simple corneal thickness, will give us a better understanding of the relationship between IOP, CCT, and optic nerve damage.

\section{References}

1. Gordon MO, Beiser JA, Brandt JD, et al. The Ocular Hypertension Treatment Study: Baseline factors that predict the onset of primary open-angle glaucoma. Arch Ophthalmol. 2002;120:714-20.

2. Leske MC, Heijl A, Hussein M, et al. Factors for glaucoma progression and the effect of treatment: the Early Manifest Glaucoma Trial.
Arch Ophthalmol. 2003;121:48-56.

3. Leske MC, Heijl A, Hyman L, Bengtsson B, Dong L, Yang Z. Predictors of long-term progression in the early manifest glaucoma trial. Ophthalmology. 2007;114:1965-72.

4. Muir KW, Jing Jin, Freedman SF. Central corneal thickness and its relationship to intraocular pressure in children. Ophthalmology. 2004;111:2220-3.

5. Dai E, Gunderson CA. Pediatric central corneal thickness variation among major ethnic populations. J AAPOS. 2006;10:22-5.

6. Evereklioglu C, Yilmaz K, Bekir NA. Decreased central corneal thickness in children with Down syndrome. J Pediatr Ophthalmol Strabismus. 2002;39:274-7.

7. Sultan G, Baudouin C, Auzerie O, De Saint Jean M, Goldschild M, Pisella PJ. Cornea in Marfan disease: Orbscan and in vivo confocal microscopy analysis. Invest Ophthalmol Vis Sci. 2002;43:1757-64.

8. Evereklioglu C, Madenci E, Bayazlt YA, Yilmaz K, Balat A, Bekir NA Central corneal thickness is lower in osteogenesis imperfecta and negatively correlates with the presence of blue sclera. Ophthalmic Physiol Opt. 2002;22:511-5.

9. Muir KW, Duncan L, Enyedi LB, Wallace DK, Freedman SF. Central corneal thickness: congenital cataracts and aphakia. Am J Ophthalmol. 2007;144:502-6.

10. Brandt JD, Casuso LA, Budenz DL. Markedly increased central corneal thickness: an unrecognized finding in congenital aniridia. Am J Ophthalmol. 2004;137:348-50.

11. Agarwal P, Devendra GG, Shetty S, Das K, Vijayalakshmi P, Muralidhar R. Central corneal thickness in congenital aniridia and its role in glaucoma in an Indian population. Asian $\mathrm{J}$ Ophthalmol. 2011;12:189-91. 